\title{
Polymer Electrophoresis across a Model Membrane
}

\author{
A. Baumgärtner, ${ }^{*} \dagger$ and J. Skolnick \\ Department of Molecular Biology, The Scripps Research Institute, La Jolla, California 92037
}

Received: May 18, 1994; In Final Form: August 9, $1994^{\circledR}$

\begin{abstract}
The translocation of a polymer driven by an external bias field of strength $\epsilon$ across a model membrane has been investigated using Monte Carlo methods. It is found that below a characteristic strength of the field $\epsilon^{*}$, the membrane is practically impenetrable. In the high-field limit, $\epsilon>\epsilon^{*}$, the permeability $\mu=\tau_{0} / \tau$, where $\tau_{0}$ and $\tau$ are the translocation times of the polymer without and in the presence of the membrane, respectively, is independent of $\epsilon$. At low fields the permeability decreases according to $\mu \sim \exp \left(-\epsilon^{*} / \epsilon\right)$. The translocation mechanism can be understood as a Kramers process describing the escape of a Brownian particle over potential barriers.
\end{abstract}

\section{Introduction}

Protein translocation across biological membranes is a basic mechanism for protein import to various cellular organelles and bacteria, ${ }^{1,2}$ for example, into mitochondria, ${ }^{3,4}$ peroxisomes, and chloroplasts ${ }^{1.5}$ and from the rough endoplasmic reticulum. ${ }^{6}$

The translocation of proteins into or across the membrane requires an input of energy. In some cases, this is provided by a transmembrane electrochemical potential and by the folding of the protein during or after its translocation mediated by a complex proteinaceous machinery. ${ }^{3,4,6}$ As a general scenario, it appears that, among others, (i) the translocation is mostly dependent on the electrochemical potential in mitochondria and in bacteria, (ii) proteins destined to be translocated are generally recognized by receptor-like components in the cytosol and on the target membrane, (iii) proteins cannot be translocated through a membrane in a tightly folded state, and (iv) many proteins enter the membrane as a precursor.

Although much progress has been made in understanding the biochemical basis of protein translocation, the physical aspects of the translocation mechanism still remain obscur.

In the present study, we consider particular aspects of the translocation process: the influence of an electric field on the transport of a polymer across a membrane. In real situations, a potential is needed for insertion of the positively charged matrix-targeting signal into the membrane (e.g. the inner membrane of mitochondria ${ }^{3}$ ), resembling a process like electrophoresis. Under the influence of an external driving field, the locomotion of the polymer through the membrane is hampered by a "barrier", which is caused by a number of factors including steric repulsions between the polymer and the lipids of the membrane, by density fluctuations of the lipids, by outof-plane fluctuations of the membrane surface, and by asymmetric fluctuations across the membrane in the case of spontaneous curvature of the membrane. ${ }^{7}$ In the present work, we are interested in effects coming from steric repulsion and from local density fluctuations of the lipids.

Translocation can be characterized by a "translocation time" $\tau$, which measures the time needed for the polymer to cross the membrane. The translocation time depends on various quantities: the strength of the electric field, the length of the polymer, the thickness of the bilayer, the lateral head group density of

\footnotetext{
* To whom correspondence should be sent.

${ }^{\dagger}$ Permanent address: Institut für Festkörperforschung, Forschungszentrum Julich, 52425 Jülich, Germany.

${ }^{\otimes}$ Abstract published in Advance ACS Abstracts, September 15, 1994.
}

the lipids, the spontaneous curvature of the membrane, etc. In the present work, we restrict our attention to the dependency of the translocation time on the strength of the external field. Since we are not interested in entanglement effects between polymer and lipids, which might be of importance for long polymers, we consider a short flexible polymer of length comparable to the width of the membrane. Using simple models for both the polymer and membrane and ignoring charge effects, we applied Monte Carlo methods to estimate the translocation times.

\section{Model and Simulation Techniques}

The Polymer Model. As the polymer model, we used the bead-spring model where $N_{\mathrm{p}}=4$ hard spheres of diameter $\sigma$ $=0.69$ are connected by harmonic springs with spring constant $K=5$ of a harmonic potential:

$$
U(x)=K\left(\ell-l_{0}\right)^{2}, l_{\min }<\ell<l_{\max }
$$

where $U\left(\ell>l_{\max }\right)=U\left(\ell<l_{\text {min }}\right)=\infty$, and $l_{\text {min }}=0.4, l_{0}=0.7$, and $l_{\max }=1.0$

Of course, the present polymer model with $N_{\mathrm{p}}=4$ beads is a fairly short chain, and the term polymer seems not to be very adequate. However, the model should be considered as a coarsegrained description of a macromolecule with mean extension comparable to the width of the membrane. In fact, the average end-to-end distance of the chain is $\langle R\rangle \approx 2.5$, which is comparable to the average width $\langle h\rangle \approx 2.7$ of the model membrane, which is described below. Assuming that both $R$ and $h$ are given in nanometers, the present models, polymer and membrane, can be considered as a reasonable coarse-grained representation of a real situation, ${ }^{8,9}$ as for example in the case of the translocation of a small protein across a membrane.

Chain dynamics is simulated by randomly displacing the spheres of the chain. Extensions of the harmonic springs are accepted according to the Metropolis scheme. A generated conformation with intersecting spheres is discarded, and the previous conformation is counted as the new one. An electric field $\mathbf{E}$ is applied perpendicular to the membrane surface. A displacement of one of the spheres from position $\mathbf{r}$ to $\mathbf{r}^{\prime}$ is accepted if

$$
\exp \left[-\mathbf{E} \cdot\left(\mathbf{r}^{\prime}-\mathbf{r}\right)\right]>\eta
$$

where $0<\eta<1$ is a random number. The strength of the field $\epsilon \equiv|\mathbf{E}|$ is measured in units of $k_{\mathrm{B}} T$. 


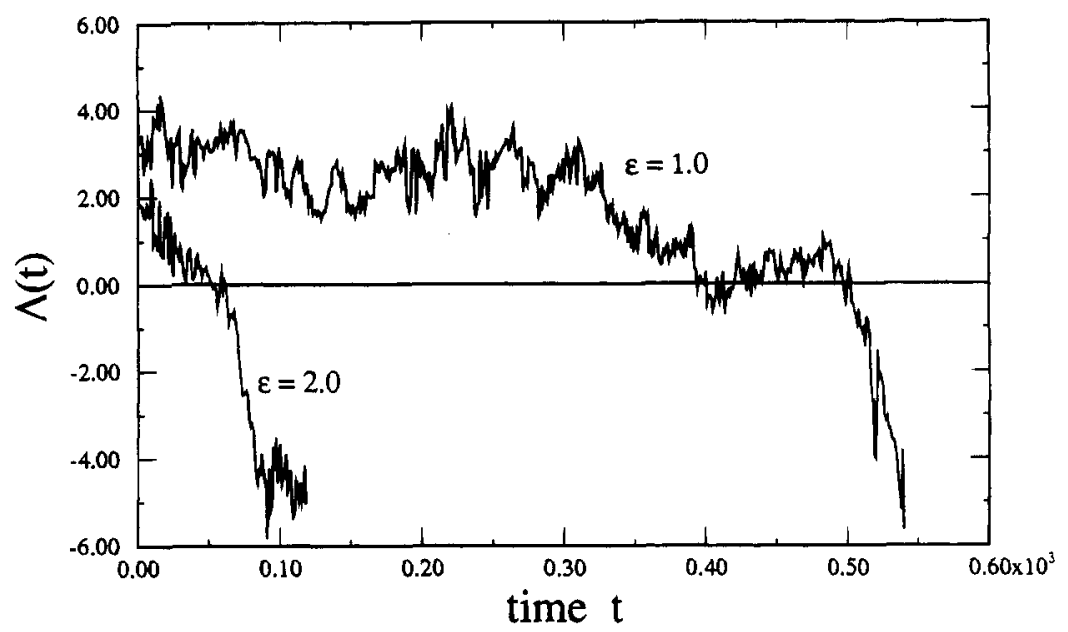

Figure 1. Penetration depth $\Lambda(t)$ versus time $t$ for field strengths $\epsilon=2.0$ and 1.0 .

The Membrane Model. The bilayer lipid membrane is simulated using a simple coarse-grained model, ${ }^{10-12}$ which consists of $N_{\mathrm{L}}$ dumbells tethered by one end to an infinitely thin penetrable interface, but are otherwise free to move from one side to the other of the interface and are allowed to diffuse in close proximity to the interface. The tether has a maximum extension of $\lambda=0.7$. Thus, each side of the lipid bilayer is comprised of dumbbells. The dumbell is a coarse-grained representation of a lipid molecule, where the size fluctuation of the lipid tail is deliberately neglected, and the mean extension of the tail is represented by a hard sphere of diameter $\sigma_{\mathrm{L}}$. For simplicity, we assume all lipids are of the same type and of cylindrical shape and, hence, design the head group of the lipid as a hard sphere of the same diameter, $\sigma_{\mathrm{L}}$, as the tail. The head and the tail are connected at fixed distance $b$. In the present model, we use $N_{\mathrm{L}}=1000, \sigma_{\mathrm{L}}=1.1$, and $b=1.32$. Since the extension of the lipid is $b+\sigma_{\mathrm{L}}$, the length to width ratio is 2.2, and hence the overall dimensions are comparable to real lipids. ${ }^{8,9}$

In the present paper, we consider the situation of a rigid interface. Preliminary studies using a "flexible" interface, ${ }^{13}$ which allows enhanced out-of-plane fluctuations of the lipids, have yielded very similar results as compared to the present work, and hence we will restrict our discussions to rigid interfaces. The geometry of the interface is a sphere of radius $R=9.373$, which has been used in order to avoid periodic boundary conditions as would occur in the case of an open membrane with free edges. The numbers of lipids in each leaflet fluctuate and are self-consistently adjusted by a flip-flop process. The average number of lipids per interfacial area in each of the two layers is $\langle\varrho\rangle \approx 0.45$. The average width of the membrane is $\langle h\rangle \approx 2.7$. The average orientation of the lipids with respect to the surface normal is $\langle\cos \theta\rangle \approx 0.77$, which is comparable to experimental values $\approx 0.7^{8}$

The dynamics of the membrane is achieved by randomly displacing the lipids. Each move is rejected if it leads to a violation of excluded volume conditions and/or tethering conditions. The displacements of polymers and lipids are performed at an equal rate. One Monte Carlo step is $2 N_{\mathrm{L}}+N_{\mathrm{p}}$ attempted moves and is defined as one time unit. Of course, the dynamics, as introduced for the membrane and polymer by means of a Monte Carlo method, cannot be compared directly to experimental results, because the Monte Carlo dynamics reflects the time evolution of the system according to master equations of conformational probability distributions rather than, as in the case of molecular dynamics simulations, the time evolution of the molecules according to Newton's equations of motion. However, for describing long time events, in many cases the two approaches give qualitatively identical results. ${ }^{14,15}$

\section{Results and Discussions}

Initially, at time $t=0$, the polymer is placed in close proximity to the outer leaflet of the membrane. The position of the center-of-mass of the polymer with respect to the interface of the bilayer is $\Lambda(t=0)>0$. The time dependence of the penetration depth $\Lambda(t)$ for two typical translocation events at two different field strengths $\epsilon$ is depicted in Figure 1. For $\Lambda$ $>0$ the polymer is located on the cis side (i.e. outside of the sphere), and for $\Lambda<0$, it is on the trans side of the bilayer. One observes that for the smaller field $\epsilon=1.0$, the polymer remains engaged with the membrane and is localized on the cis side longer than in the case of the larger field $\epsilon=2.0$. The "translocation time" $\tau$ of the polymer can be defined as the time the polymer needs to completely cross the membrane, from $\Lambda(0)>0$ to $\Lambda(\tau)<0$. Of course, the choice of the traveled distance

$$
\Delta=\Lambda(0)+|\Lambda(\tau)|
$$

is in principle arbitrary. However, since we are interested mainly in effects coming from the membrane, we have to define $\Delta$ such that only within this interval the polymer is in contact with lipids, and therefore it should exclude contributions from free draining effects well outside of the membrane.

Since the polymer has an average extension of $\langle R\rangle \approx 2.5$ and the maximum width of one leaflet of the bilayer is 3.12 , one can assume that the polymer is virtually disengaged with the membrane at a distance $\Lambda(\tau)=-5.5$. Initially the polymer is placed at $\Lambda(0)=5.0$.

In order to obtain an ensemble average over various translocation events, several Monte Carlo runs with different starting points have been performed. In most cases, an average over 13 different starts are sufficient in order to obtain a mean deviation of $\sqrt{ } \Sigma_{i}\left(\tau_{i}-\tau\right)^{2} / \tau<0.2$. Estimates of average translocation times $\tau$ at various $\epsilon$ are presented in Figure 2 .

These translocation times have to be compared with the "unperturbed" translocation time $\tau_{0}$, in which the polymer needs to travel the same distance $\Delta$ and starting at the same places $\Lambda(0)$ but without the presence of the membrane. The Monte Carlo results are shown in Figure 2. In order to support the reliability of the simulation results, it is useful to compare them to standard Brownian theory, which requires

$$
\Delta^{2}=D_{0} \tau_{0}+E_{0}{ }^{2} \tau_{0}{ }^{2}
$$




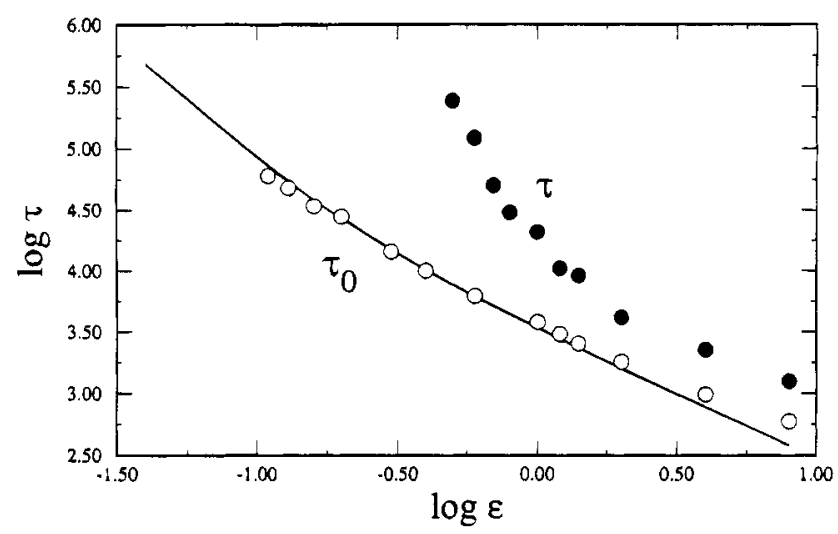

Figure 2. Transition times $\tau$ and $\tau_{0}$ as function of field strength $\epsilon$. The full curve corresponds to eq 5 with $D_{0}=0.003$ and $E_{0}=0.002 \epsilon$.

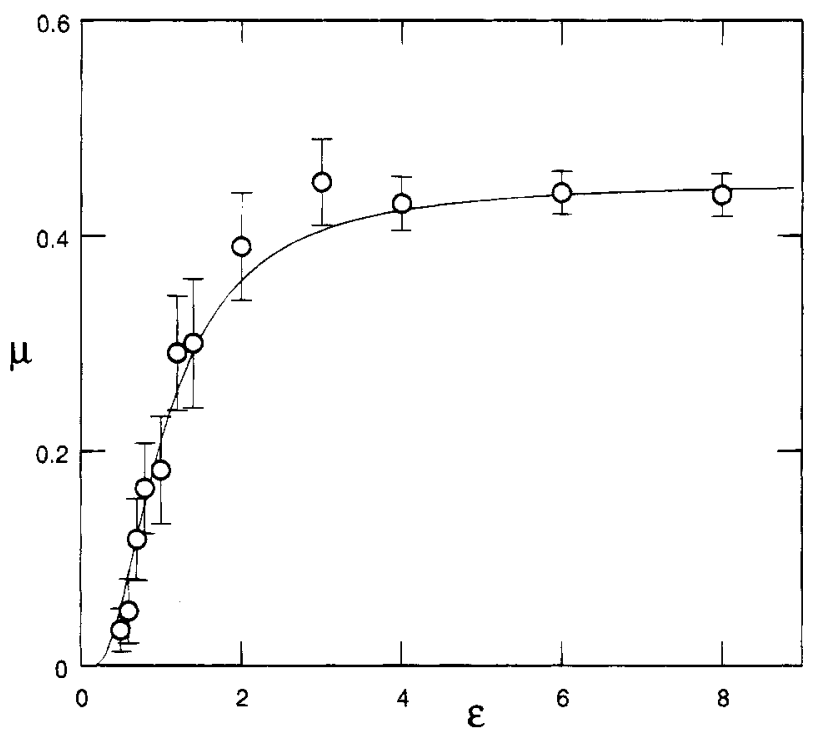

Figure 3. Permeability $\mu=\tau_{0} / \tau$ versus field strength $\epsilon$. The line corresponds to eq 8 with $\epsilon^{*}=1.5$ and $\mu_{\infty}=0.45$.

where the first term corresponds to the Brownian motion and the second term to the continuous drift under the influence of the field, and the coefficients $D_{0}$ and $E_{0}$ are effective diffusion and field coefficients, respectively. (The factor $1 / 2$ supposedly appearing in the first term of eq 4 according to the usual Einstein relation has been incorporated in the definition of $D_{0}$.) Equation 4 can be rewritten as

$$
\tau_{0}=\frac{D_{0}}{2 E_{0}^{2}}\left(1+\sqrt{ } 1+\left(2 \Delta E_{0} / D_{0}\right)^{2}\right)
$$

Using estimates of $D_{0} \approx 0.003$ and $E_{0} \approx 0.002 \epsilon$, eq 5 is in reasonable agreement with the Monte Carlo data as shown in Figure 2. In the range of fields $0.1 \leq \epsilon \leq 10.0$, which is of interest in the present considerations, eq 5 is approximately

$$
\tau_{0} \approx A_{0} \epsilon^{-1}
$$

where $A_{0}=500 \Delta$. The permeability of the membrane can be defined as

$$
\mu=\tau_{0} / \tau
$$

This quantity is depicted in Figure 3. At large fields the permeability saturates and becomes a constant, $\mu_{\infty} \approx 0.45$. This constant is not universal and may depend on various parameters such as the density of lipids and curvature of the membrane.
For $\epsilon<\epsilon^{*} \approx 1.5$, the permeability rapidly decreases to 0 . From our data we cannot decide whether the membrane becomes strictly impenetrable (i.e. $\mu=0$ ) below a critical value $\epsilon_{\mathrm{c}}$ with $0<\epsilon_{\mathrm{c}}<\epsilon^{*}$. This would imply that the translocation time $\tau$ exhibits a singular behavior, $\tau \rightarrow \infty$ for $\left|\epsilon-\epsilon_{\mathfrak{c}}\right| \rightarrow 0$.

Empirically, we found that the permeability is fairly well described by

$$
\frac{\mu}{\mu_{\infty}}=\frac{1}{\cosh \left(\epsilon^{*} / \epsilon\right)}
$$

which is depicted in Figure 3. For the limiting cases one has at large fields

$$
\frac{\mu}{\mu_{\infty}} \approx 1-\frac{1}{2}\left(\frac{\epsilon^{*}}{\epsilon}\right)^{2} \quad\left(\epsilon^{*} / \epsilon<1\right)
$$

whereas at low fields

$$
\frac{\mu}{\mu_{\infty}} \approx 2 \exp \left(-\epsilon^{*} / \epsilon\right) \quad\left(\epsilon^{*} / \epsilon>1\right)
$$

However, eq 8 implies that in the zero-field limit the permeability is 0 . Of course, this cannot be correct in a strict sense since there always exists a very small probability that the chain will spontaneously cross the repulsive barrier caused by the membrane. Therefore, eq 8 cannot be considered to be rigorous, but should be considered as a good approximation. In fact, an estimate of the translocation time in the zero-field limit using Monte Carlo methods yields $\tau>10^{8}$ and $\tau_{0} \approx 10^{4}$, and hence $\mu \approx 10^{-4}$, which means that the membrane is practically impenetrable at zero field. For comparison, the smallest permeability as measured by Monte Carlo methods in the presence of the field and depicted in Figure 3 is $\mu \approx 0.033$ at $\epsilon=0.5$.

The interpretation of the polymer electrophoresis across a fluctuating membrane can be related to the Kramers problem. ${ }^{16-18}$ According to the Kramers criterion for a Brownian particle to escape over potential barriers, the average transition time is given by

$$
\tau \sim \exp [V(\Delta)]
$$

where $V(\Delta)$ is the maximum barrier height within the distance $\Delta$ the particle has traveled. Comparing this result with the translocation time in the case of low fields as obtained using eqs 6,7 , and 10 ,

$$
\tau \sim \epsilon^{-1} \exp \left(\epsilon^{*} / \epsilon\right)
$$

leads to the conclusion that $V \sim \epsilon^{*} / \epsilon$.

It is interesting to note that the translocation across a "frozen" membrane consisting of immobile lipids follows a different mechanism than in the case of "fluid" membranes. The locomotion of the polymer across such a "frozen" membrane stresses the importance of the fluctuations of the lipids. Simulations at various realizations of frozen orientations and locations of lipids in the membrane show that for large fields, $\epsilon>1$, the translocation time is essentially constant, $\tau_{\mathrm{f}} \approx 10^{5}$, but about 2 orders of magnitude larger than in the case of mobile lipids. For small fields, $\epsilon<0.8$, the frozen membrane is practically impenetrable, $\tau_{\mathrm{f}} \rightarrow \infty$; that is translocation events were not observed for times $t<10^{7}$. The observations at large fields indicate that the distribution of "permanent holes" as created randomly in the frozen membrane essentially control the translocation process in this case, and hence the translocation time is constant. The observations at small fields can be 
interpreted such that a translocation event becomes highly unlikely with decreasing field strength due to the steric repulsion between membrane and polymer, which dominates the polymer motion and hence prevents the polymer from finding a hole in the membrane.

\section{Conclusions}

The present investigations aimed to support our understanding of the physical effects during the electrophoresis of a short flexible macromolecule across a fluid membrane. The basic conclusion is that the translocation is restricted by a barrier due to the positional and orientational fluctuations of the lipids, and the process resembles the Kramers problem involving the escape of a Brownian particle over repulsive potentials. In particular, it is found that in the high-field limit, $\epsilon>\epsilon^{*}$, the permeability $\mu=\tau_{0} / \tau$ is independent of $\epsilon$, whereas at low fields the permeability decreases according to $\mu \sim \exp \left(-\epsilon^{*} / \epsilon\right)$.

Many aspects of the translocation process have been deliberately neglected for the sake of clarity. Several questions now have to be addressed, as there are effects coming from entanglements between long polymers and lipids during electrophoresis, ${ }^{19}$ from the entropy-controlled partitioning of long polymers into membranes, ${ }^{20}$ and from fluctuation-induced bias across curved membranes. ${ }^{7}$ Moreover, effects coming from types of interactions between lipids and polymers other than pure repulsive ones, as considered in the present work, are of interest, in particular with regard to real experimental situations.
Acknowledgment. We would like to thank A. Kolinski for useful comments. Support by the Joseph Drown Foundation is gratefully acknowledged. This research was also supported in part by NIH Grant GM 38794. The simulations were performed on the IBM ES-9000 at the Forschungszentrum Jülich.

\section{References and Notes}

(1) Wickner, W. T.; Lodish, H. F. Science 1985, 230, 400.

(2) Singer, S. J.; Maher, P. A.; Yaffe, M. P. Proc, Natl. Acad. Sci. $1985,84,1015$.

(3) Schatz, G. Protein Sci. 1993, 2, 141.

(4) Wienhues, U.; Neupert, W. BioEssays 1992, 14, 17.

(5) Theg, S. M.; Scott, S. V. Trends Cell Biol. 1993, 3, 186.

(6) High, S. BioEssays 1992, 14, 535.

(7) Baumgärtner, A.; Skolnick, J., submitted.

(8) Israelachvili, J. M.; Marcelja, S.; Horn, R. G. Q. Rev. Biophys. $1980,13,121$.

(9) Gennis, R. B. Biomembranes: Molecular Structure and Function, Springer: New York, 1989.

(10) Evans, E. A. Biophys. J. 1974, 14, 923.

(11) Petrov, A. G.; Bivas, I. Prog. Surf. Sci. 1984, 16, 389.

(12) Baumgärtner, A. J. Chem. Phys., in press.

(13) Baumgärtner, A.; Ho, J. S. Phys. Rev. A 1990, 41, 5747. Baumgärtner, A. J. Chem. Phys. 1993, 98, 7496.

(14) Verdier, P. H.; Stockmayer, W. H. J. Chem. Phys. 1962, 36, 227.

(15) Rey, A.; Skolnick, J. Chem. Phys. 1992, 158, 199.

(16) Kramers, H. A. Physica 1940, 7, 284.

(17) Chandrasekhar, S. Rev. Mod. Phys. 1943, 15, 1.

(18) De Gennes, P. G. J. Stat. Phys. 1975, 12, 463.

(19) Zimm, B.; Levene, S. D. Q. Rev. Biophys. 1992, 25, 171

(20) Muthukumar, M.; Baumgärtner, A. Macromolecules 1989, 22, 1937. 